\title{
Electronically driven fragmentation of Ag nanocrystals revealed by ultrafast electron crystallography
}

\author{
Ramani K. Raman, Ryan A. Murdick, Richard J. Worhatch, Yoshie \\ Murooka, Subhendra D. Mahanti, Tzong-Ru T. Han, and Chong-Yu Ruan* \\ Physics and Astronomy Department, Michigan State University, East Lansing, Michigan 48824-2320
}

We report a ultrafast electron diffraction study of silver nanocrystals under surface plasmon resonance excitation, leading to a concerted fragmentation. By examining simultaneously transient structural, thermal, and Coulombic signatures of the prefragmented state, an electronically driven nonthermal fragmentation scenario is proposed.

PACS numbers: $36.40 . \mathrm{Gk}, 73.22 . \mathrm{Lp}, 78.67 . \mathrm{Bf}, 61.05 . \mathrm{J}-$

The ability to image defect growth processes is central to the understanding of the electronically induced structural phase transitions in solids [1 3$]$. Whereas optical and photoemission studies have provided significant insights into the initial electronic processes that are strongly coupled to lattice degrees of freedom, the mechanism bridging the femtosecond (fs) optical seeding to the picosecond(ps)-to-nanosecond(ns) macroscopic structural changes remains a central topic to be elucidated. Recent developments in ultrafast diffraction techniques have enabled direct probing of atomic dynamics and helped accentuate the important role of electronic excitation in initiating coherent motions [4], bond dilation [5, 6] and structural transformations [7 9$]$. Here, employing ultrafast electron nanoscale crystallography [10], we demonstrate a direct structural study of spatially inhomogeneous processes in $\mathrm{Ag}$ nanocrystals (NCs) induced via surface plasmon resonance (SPR) excitation. Contrary to an impulsive process leading to fragmentation, we find that the dominant dynamical feature in the prefragmentation stage is a defect-mediated instability growth, creating sub-nanocrystalline domains with hot surface and relatively cold core. Electronic effects are proposed to account for the incipient creation and subsequent growth of lattice inhomogeneities directly responsible for fragmentation, which are corroborated by the evidences of correlated charge localization and defect percolation on the picosecond timescale following photoexcitation.

Photoinduced fragmentation of NCs has been a subject of recent intense interests, with a range of results favoring either thermal ablation [11, 12], or a nonthermal pathway involving charging of the nanoparticles $13-16$ or creation of strong fields [17]. The mechanism for photoinduced structural changes appears to depend on the pulse duration [18], with the surrounding solvent limiting the thermal dissipation [19]. Using ultrafast electron crystallography (UEC) [10], we have directly probed the transient structures of the NCs up to the ablation limit, and identified the transient dynamical structures and the charge states. In contrast to earlier colloidal suspensions studies, this experiment is conducted in vacuum
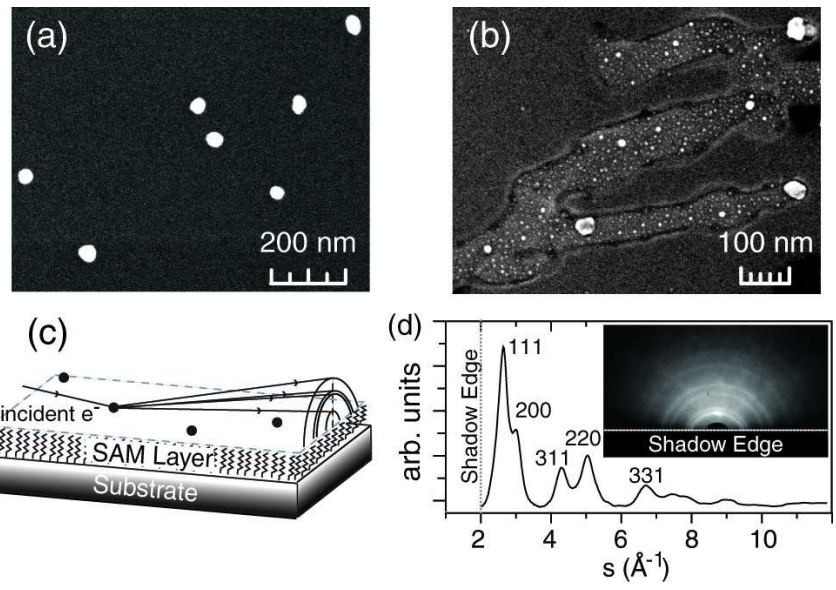

FIG. 1: (a) SEM images of $40 \mathrm{~nm} \mathrm{Ag}$ NC sample before laser irradiation and (b) after irradiation (400 nm, $F=22$ $\mathrm{mJ} / \mathrm{cm}^{2}$ ), showing fragmentation. (c) Schematics of diffraction/sample geometry employed in this study. (d) Ground state 1D structure function obtained from the 2D powder diffraction pattern (inset), showing characteristic fcc peaks.

with sufficient dispersion of nanoparticles to avoid longrange surface plasmon induced interaction and solvent effect. Colloidal silver NCs (Ted Pella, $40 \pm 7 \mathrm{~nm}$ ) are dispersed ex-situ on a silicon substrate functionalized with a self-assembled monolayer (SAM) of alkane-silane ((3Aminopropyl)trimethoxysilane or APTMS, Ted Pella) molecules that suppress substrate signals. SEM characterization of the sample showed uniform coverage, with low mean areal density of $7 \mathrm{NC}$ per $\mu m^{2}$ (Fig. 1(a)). First, the NCs are irradiated using ultrafast laser pulse ( $50 \mathrm{fs}, p$-polarized, $45^{\circ}$ incidence) at $400 \mathrm{~nm}$, which is within their SPR bandwidth $(385 \pm 40 \mathrm{~nm})$, to check for traces of fragmentation. At a critical fluence $\mathrm{F}=22$ $\mathrm{mJ} / \mathrm{cm}^{2}$ (multi-shot) SPR excitation leads to fragmentation into predominantly $2 \mathrm{~nm} \mathrm{NCs,}$ as shown in Fig. 1(b). Prior to such laser excitation, the UEC patterns obtained from these randomly oriented NC samples resemble a 'powder diffraction' pattern, as shown in Fig. 1(c). The 1D structure function deduced via a radial averaging of these 2D patterns [10] displays clear fcc symmetry 

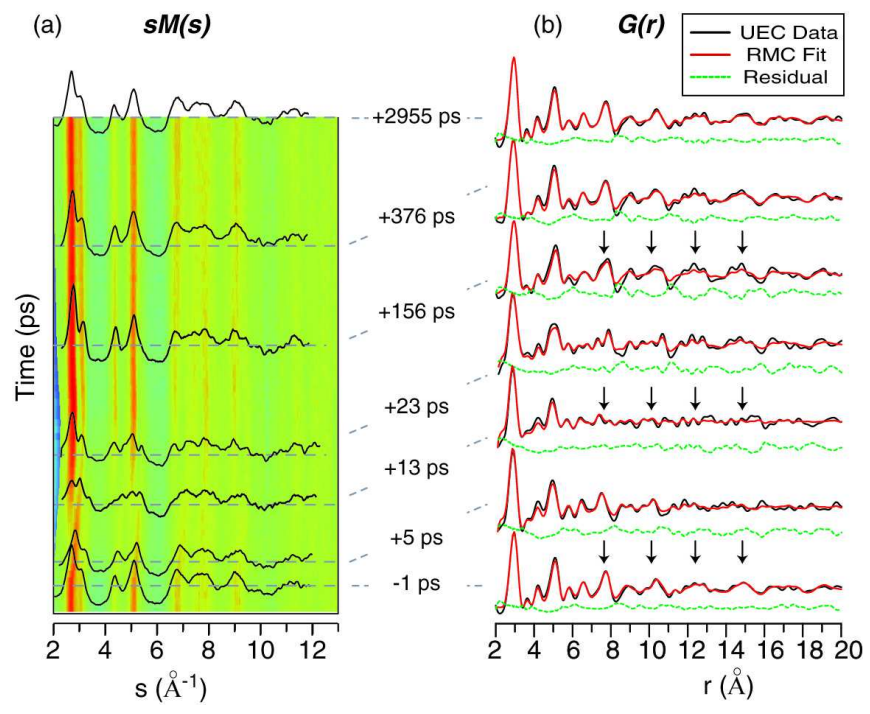

FIG. 2: (Color online) UEC Data. (a) Time evolution map of the normalized structure factor $s M(s)$ of $\mathrm{Ag}$ NCs excited at SPR at $F=17 \mathrm{~mJ} / \mathrm{cm}^{2}$, with $s M(s)$ at select times explicitly overlayed. (b) Pair correlation function $G(r, t)$, corresponding to the select times in (a). The arrows point to the medium range order peaks, which completely dephase by $t=+13 \mathrm{ps}$, before their eventual revival at long times.

(Fig. 1(d). We subsequently probe the transient structures and the atomic rearrangements using laser fluence just below the critical fragmentation fluence. Sample integrity was retained during pump-probe cycles of UEC, as confirmed by SEM imaging following the conclusion of experiment.

The gross features of photoinduced structural changes are evident from the transient alteration of the normalized structure function [10] $s M(s)$, ( $s$ is the electron scattering wave-vector) obtained at the prefragmenting fluence $F=17 \mathrm{~mJ} / \mathrm{cm}^{2}$, shown in Fig. 22(a). By $t=+13 \mathrm{ps}$, the initial fcc ordered diffraction maxima diminish while the nearby diffusive scattering gain strength and grow into multiple peaks, indicating a departure from the cubic symmetry. The reduction of long-range order is evident from the corresponding pair correlation function $G(r, t)$ [10], obtained via a Fourier analysis of the diffraction patterns, as shown in Fig. 2(b). The atom-atom correlation peaks greater than $10 \AA$ in $G(r, t)$ smear out completely by $t=+13 \mathrm{ps}$, whereas the short-range peaks $(r \leq 10$ $\AA)$ retain their strength, thereby indicating the persistence of short-range order during the pre-fragmentation process.

To identify the origins of this structural disorder, we first calculate from each Bragg intensity a parameter $W=\left(1 / s^{2}\right) \ln \left(I / I_{0}\right) . W$ is closely related to the meansquare displacement of atoms $\left\langle\Delta \bar{u}_{\perp}^{2}\right\rangle\left(4 \mathrm{~W}=\left\langle\Delta \bar{u}_{\perp}^{2}\right\rangle\right)$ - a tool often used in cases of quasi-equilibrium conditions to extract lattice temperature via the Debye-Waller relation. In such cases, $\left\langle\Delta \bar{u}_{\perp}^{2}\right\rangle$ is independent of the diffrac-
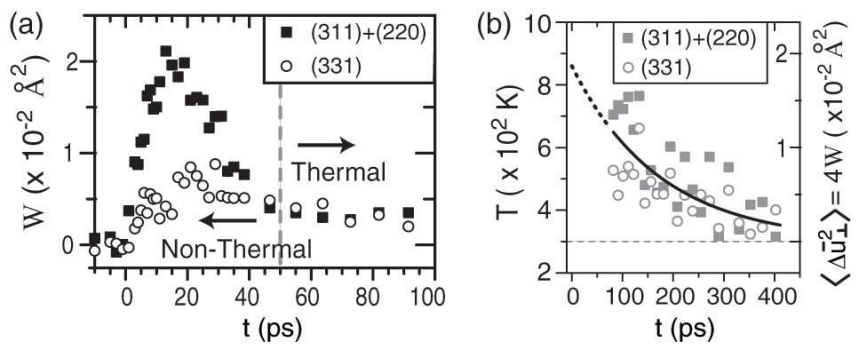

(c)
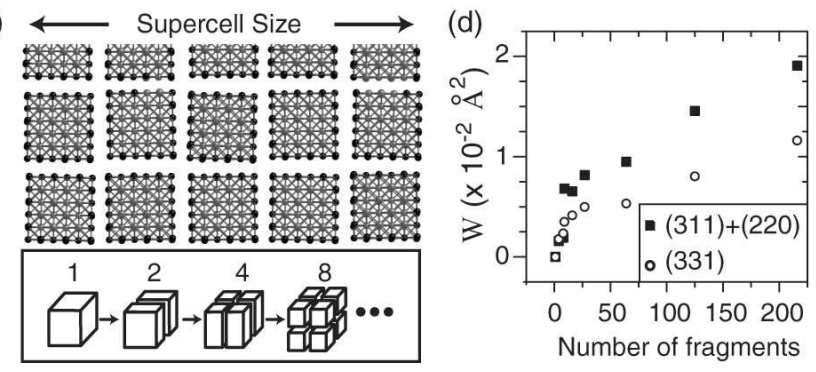

FIG. 3: Thermal signatures of fragmentation process. (a) $W=\left(1 / s^{2}\right) \ln \left(I / I_{0}\right)$ determined from intensity drop of diffraction rings and (b) corresponding lattice temperature deduced at long times, with exponential fit (solid line) to data beyond $t>80 \mathrm{ps}$. Early time data are excluded for clarity. See [21]. (c) Planar slice of the fragmented supercell. Random relative orientations imposed amongst the fragments removes inter fragment correlation. Black atoms indicate undercoordinated sites. The cartoon depicts the fragmentation scheme. (d) Variation of simulated $W$ with the number of fragments.

tion order used [20]. However, we find here (Fig. 3(a)) that for $t<50 \mathrm{ps}, W$ determined from higher order (331) peak is smaller than that from the lower order (311) and (220) peaks, indicating the nonthermal nature of the structure disordering process. We will argue that this anisotropy in $W$ is the manifestation of a unique structural change that destroys the long-range coherence within NCs acting as a precursor to the fragmentation observed above the critical fluence. At longer times $(t>50 \mathrm{ps}), W$ shows no $s$-dependence, thus allowing us to reliably extract the lattice temperature from $W$ [20], shown in Fig. 33(b). The temperature decay is in good agreement with an interface limited thermal relaxation model based on thermal conductivity of the SAM 21] and suggests a temperature rise in NCs of at most $860 \pm 150 \mathrm{~K}$ at early times, which is insufficient to cause thermal ablation. To capture the essence of this nonthermal transformation, we carry out a simulation of diffraction signatures expected from NC fragmentation. Starting from a $15 \times 15 \times 15$ supercell structure for the NC, we fragment (subdivide) the supercell into smaller units as illustrated in Fig. 3(c). The separation between fragments and their relative orientations is altered randomly so as to remove any inter fragment correlations. Such fragmented assemblies are generated for several fragmentation numbers, following which, the $s M(s)$ intensity from the fragmented assembly is computed for each case. The 
lattice temperature is maintained (at $300 \mathrm{~K}$ ), so that any changes evident in the $s M(s)$ intensities arise solely from the fragmentation process. The $W$ parameter extracted from these 'simulated' $s M(s)$ is shown in Fig. 3(d), and exhibits the same anisotropy trend seen in the experiment. A qualitative agreement in the scale of anisotropy seen in the experiment and simulation is reached when the number of fragments exceed 100, corresponding to an individual fragment size of $\approx 2 \mathrm{~nm}$, which is close to the average fragment sizes observed in the SEM image (Fig.1(b)). These results indicate that a nonthermal restructuring (prefragmentation) rather than a thermal disorder better explains the experimental observations.

To find out the role of Coulombic forces in the fragmentation of the NCs, we examine two relevant processes leading to charging of the NCs: photoemission and charge transfer between the NCs and the supporting surface. To determine the photoemission yield, we use a projection imaging method [22] illustrated in Fig. 4(a,b), where surface scattered electrons originating from point $\mathrm{P}$ intercept the laser-induced vacuum emitted charge cloud, thus casting its shadow on the CCD. By analyzing the normalized shadow images of the electron cloud, we can extract the density of photoemitted electrons, which we find to double (to $7.9 \times 10^{7} \mathrm{e} / \mathrm{cm}^{2}$ ) in the presence of NCs relative to that from a bare Si surface, as evident from Fig. 4(c). This enhancement is significant considering the NCs occupy less than $1 \%$ of the surface. Similar photoemission enhancement from Ag NCs has been observed in other studies as well and attributed to SPR assisted multiphoton processes [23]. In addition to this, we observe another competing, SPR enhanced localized charge transfer channel between NCs and the substrate, that causes even more pronounced charging of the NCs. We quantify this process via a diffractive voltammetry approach [10, 22, 24] as illustrated in Fig. 4(d). Recall that the Ag NCs sit on top of a SAM layer, which produces its own diffraction signal in the form of a strong (001) peak at $s=2.75 \AA^{-1}$. Any interfacial charge transfer between the $\mathrm{Ag} \mathrm{NC}$ and the substrate leads to the creation of a transient field at the $\mathrm{Ag} \mathrm{NC} / \mathrm{SAM} / \mathrm{Si}$ interface, causing a refraction shift of probing electron beam traversing the region in between. Thus, from the shift of the (001) SAM peak, we numerically compute the corresponding interfacial field $E$ (and potential $\Delta V$ ). Based on an interfacial capacitance $\mathrm{C}=7.5$ attofarad, we thus determine a maximum average charging per $\mathrm{NC}$ of $q \approx 400 \mathrm{e}^{+}$, which is in contrast to $0.2 \mathrm{e}^{+}$estimated from the vacuum emission measurement. This corresponds to a Coulomb fissility ratio $X=q^{2} / n \approx 0.08$, where $n \approx 2 \times 10^{6}$ is the number of atoms within the $\mathrm{NC}$, which is below the direct Coulomb fissility regime $(0.3<X<1)[16]$.

Our observation is consistent with a progressive Coulomb-induced fragmentation scenario proposed by Kamat and coworkers [13]. The transient charging curve
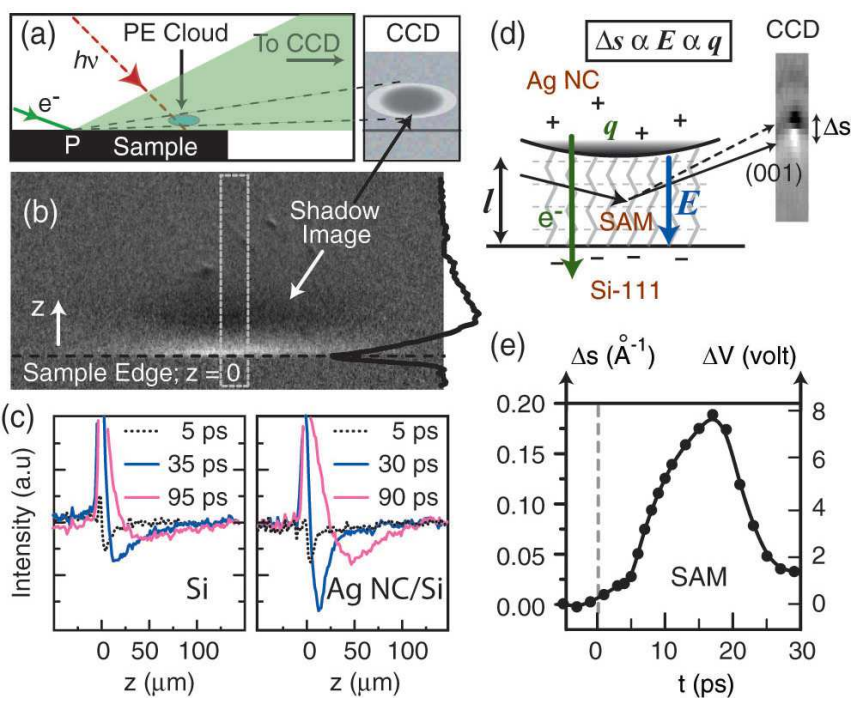

FIG. 4: (Color Online) Charge signatures of fragmentation process. (a) Point-projection imaging of vacuum emitted photoelectrons (PE). (b) Normalized shadow images of the PE cloud. The cloud profile is shown on the right. (c) Evolution of $\mathrm{PE}$ cloud profiles over Si-111 surface with and without the $\mathrm{Ag} \mathrm{NCs}$, showing significant enhancement in presence of $\mathrm{Ag}$ NCs. (d) Concept of diffractive voltammetry. (e) Shift of the SAM (001) peak with time and corresponding interfacial potential $\Delta V=E \cdot l$, using $l=1 \mathrm{~nm}$.

(Fig. 4(e)) shows an initial 6 ps incubation period for charge redistribution at $\mathrm{NC}$ interface which is absent at non-SPR excitations [10], and is thus indicative of transient trapping of positive charges at the excitation sites. The migration of charges to the interface and the accompanied rise of interfacial field (Fig. 4(e)) is hindered as the lattice is undergoing atomic restructuring during this incubation period, which also coincides with the period of $W$ - anisotropy (Fig [3(a)).

The observed correlation between the atomic process and charge trapping suggests that local valence instabilities which cause bond softening [6] lead to structural defects and charge localization as seen in our studies. Earlier studies of fs laser induced melting in metals using optical and photoemission techniques have revealed that interband transitions [25] and thermionic emission [26] can both lead to rapid bond softening within the initial non-equilibrium time scales of core hole lifetime $(\approx$ $100 \mathrm{fs}$ ) and electron-phonon coupling time ( $\approx 1 \mathrm{ps})[27]$. In $\mathrm{Ag}$, the nonlinear interband excitation involving inner valence shell $(d \rightarrow s p)$ is found to be strongly coupled to the SPR dephasing pathway 28]. These excitations can provide seeds for valence destabilization through SPR. Such a process would be inhomogeneous in nature and proceed on a phonon timescale.

To visualize transient atomic processes associated with electronic excitation, we employ a Reverse Monte Carlo (RMC) sturcture refinement scheme [10]. While the ear- 

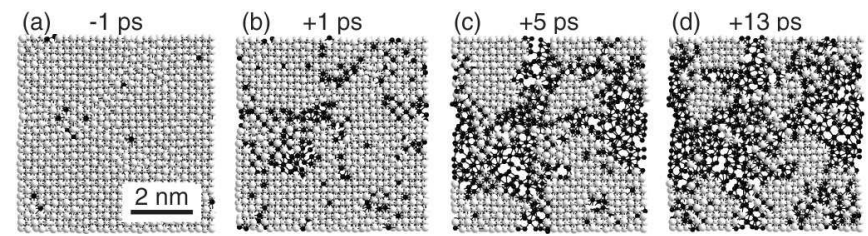

FIG. 5: Prefragmentation states of $\mathrm{Ag} \mathrm{NC}$ at select times, determined using the structure refinement scheme described in the text. Growth of local disorder is apparent from the increase in number of undercoordinated atoms (black)

lier fragmentation simulation yielded qualitative insights into the origin of observed $W$ - anisotropy, the approach here seeks to refine/adjust the local atomic positions within the $15 \times 15 \times 15 \mathrm{Ag}$ supercell so as to fit both the positions and intensities of $G(r, t)$ peaks generated from these structures to those determined experimentally at each time instance, as shown in Fig.2(b). First, we confirm that the data has sufficient signal-to-noise ratio since the RMC result of the ground state $(t<0)$ retains a robust fcc sturcture, as shown in Fig. 5(a). Given that the diffraction changes between consecutive time frames are small, the refinement scheme progressively tracks the disordering process from one time instance to the next, while being constrained by the correlation between atomic positions in neighboring time frames that is inherently present in the diffraction patterns. Selected RMC results are depicted in Figs. 5(a)-(d) with a planar slice cut off from the 3D supercell to expose the degradation of the pristine fcc lattice over time. The atomic sites colored in black represent topological defects with coordination number $\mathrm{N}_{c} \leq 10$, whereas the fully coordinated ones have $\mathrm{N}_{c}=12$ corresponding to the fcc structure. We find that these defects, which are initially sparsely distributed following SPR, visibly grow and percolate into strips in $10 \mathrm{ps}$ and then saturate, leaving behind fragmented nanocrystalline domains with an average size $\approx 2 \mathrm{~nm}$ [21]. This disordered state persists for nearly $10 \mathrm{ps}$ before a slow recovery to fcc on a time scale $\approx 100$ ps. Further analysis shows that among those undercoordinated sites the nearest neighbour distance exhibits large fluctuations indicating bond-softening in this region [6] characterized by their averaged root-mean-square displacements on the order of $0.15 \AA$, which is 4 times higher than those in the region of core domains.

In summary, an electronically driven, progressive fragmentation of $\mathrm{Ag} \mathrm{NCs}$ is presented. We suggest that the fragmentation process is triggered by the creation of local valence instabilities, caused by redistribution of local charge density, facilitated in Ag through the strong nonlinear coupling between SPR and interband transition. When sufficient valence instabilities are instigated at a high fluence $\left(F \geq 17 \mathrm{~mJ} / \mathrm{cm}^{2}\right)$, the electronic states are strongly perturbed, leading to a prolonged ps lifetime of the local charge redistribution associated with valence ex- citation due to an insufficient dynamical screening. Such a dynamical localization feature is central to the creation and growth of the topological defects, which can persist on the phonon timescales, and could be common in nonequilibrium photoinduced structural phase transition.

We thank P.M. Duxbury, S.J.L. Billinge, R. Shen, and F. Vallée for critical discussions. The experimental work was supported by Department of Energy under Grant DE-FG02-06ER46309. The theoretical analysis was supported (R.J.W, R.A.M) by National Science Foundation under Grant DMR-0703940.

* Email: ruan@pa.msu.edu

[1] K. Nasu, Photoinduced phase transitions (World Scientific, Hackensack, 2004).

[2] E. Collet, et al., Science 300, 612 (2003).

[3] M. M. Qazilbash, et al., Science 318, 1750 (2007).

[4] M. Bargheer, et al., Science 306, 1771 (2004).

[5] P. Baum, D.-S. Yang, and A. H. Zewail, Science 318, 788 (2007).

[6] D. M. Fritz, et al., Science 315, 633 (2007).

[7] C.-Y. Ruan, et al., Science 304, 5667 (2004).

[8] N. Gedik, et al., Science 316, 425 (2007).

[9] B. J. Siwick, J. R. Dwyer, R. E. Jordan, and R. J. D. Miller, Science 302, 1382 (2003).

[10] C.-Y. Ruan, et al., Microsc. Microanal. 15, 323 (2009), arXiv:0910.4407v1.

[11] A. Takami, H. Kurita, and S. Koda, J. Phys. Chem. B 103, 1226 (1999).

[12] S. Inasawa, M. Sugiyama, and Y. Yamaguchi, J. Phys. Chem. B. 109, 3104 (2005).

[13] P. V. Kamat, M. Flumiani, and G. V. Hartland, J. Phys. Chem. B 102, 3123 (1998).

[14] S. Link, C. Burda, B. Nikoobakht, and M. A. El-Sayed, J. Phys. Chem. B 104, 6152 (2000).

[15] P. L. Redmond, X. Wu, and L. Brus, J. Phys. Chem. C 111, 8942 (2007).

[16] H. Muto, K. Miyajima, and F. Mafune, J. Phys. Chem. C 112, 5810 (2008).

[17] A. Plech, V. Kotaidis, M. Lorenc, and J. Boneberg, Nat. Phys. 2, 44 (2006).

[18] C. B. Schaffer, A. Brodeur, and E. Mazur, Meas. Sci. Tech. 12, 1784 (2001).

[19] G. V. Hartland, Phys. Chem. Chem. Phys. 6, 5263 (2004).

[20] R. K. Raman, et al., Phys. Rev. Lett. 101, 077401 (2008).

[21] See EPAPS Document No. [to be assigned] for details.

[22] R. K. Raman, Z. Tao, T.-R. Han, and C.-Y. Ruan, Appl. Phys. Lett. 95, 181108 (2009).

[23] J. Lehmann, et al., Phys. Rev. Lett. 85, 2921 (2000).

[24] R. A. Murdick, R. K. Raman, Y. Murooka, and C.-Y. Ruan, Phys. Rev. B 77, 245329 (2008).

[25] C. Guo, G. Rodriguez, A. Lobad, and A. J. Taylor, Phys. Rev. Lett. 84, 4493 (2000).

[26] P. Grua, et al., Phys. Rev. B 68, 035424 (2003).

[27] N. Del Fatti, et al., Phys. Rev. B 61, 16956 (2000).

[28] C. Voisin, N. D. Fatti, D. Christofilos, and F. Vallee, J. Phys. Chem. B 105, 2264 (2001). 